04

\title{
Динамика поля в резонаторе с медленно осциллирующим металлическим зеркалом
}

\author{
() Л.А. Нестеров ${ }^{1}$, Н.А. Веретенов ${ }^{1,2}$, Н.Н. Розанов ${ }^{1,2,3 \mp}$ \\ ${ }^{1}$ Университет ИТМО, \\ 197101 Санкт-Петербург, Россия \\ ${ }^{2}$ Государственный оптический институт им. С.И. Вавилова, \\ 199053 Санкт-Петербург, Россия \\ ${ }^{3}$ Физико-технический институт им. А.Ф. Иофрфе, \\ 194021 Санкт-Петербург, Россия \\ ฯ e-mail: nnrosanov@mail.ru
}

Поступила в редакцию 04.02.2018 г.

\begin{abstract}
Аналитически исследовано поведение электромагнитного поля в резонаторе, у которого одно из металлических зеркал неподвижно, а второе осциллирует в окрестности своего положения равновесия. При этом рассматривается случай, когда период осцилляций зеркала существенно превосходит характерное время полного обхода резонатора электромагнитным излучением. Для решения задачи использован метод двух временных масштабов. Метод позволяет решить задачу с помощью разложения по малому параметру, которым является отношение времени обхода резонатора к периоду осцилляций зеркала. Получено и исследовано решение задачи в нулевом приближении по малому параметру. Это решение является корректным вплоть до амплитуд колебаний зеркала порядка средней длины резонатора. Показано, что рассматриваемая схема может быть использована для усиления и генерации электромагнитного излучения. Проведено сопоставление полученных результатов для осциллирующего зеркала с результатами точного решения задачи, когда зеркало движется равномерно и прямолинейно. Показано, что в обоих случаях изменение полной энергии поля в резонаторе обратно пропорционально текущему значению длины резонатора.
\end{abstract}

DOI: $10.21883 /$ OS.2018.06.46089.33-18

\section{Введение}

Исследования динамики электромагнитного поля в резонаторах с подвижными зеркалами проводились на протяжении последних 50 лет. С одной стороны, изучались классические параметрические волновые явления в таких системах (см. [1], а также [2-4] и список цитированной там литературы). С другой стороны, исследования использовались для анализа квантового эффекта Казимира (см. обзор работ в [5]). Проблема имеет как принципиальное научное, так и прикладное значение в связи с возможностью преобразования кинетической энергии движущихся зеркал в энергию электромагнитного излучения.

В настоящей работе также рассматривается чисто классическая задача о динамике электромагнитного поля в резонаторе с неподвижным металлическим зеркалом, расположенным в начале координат и аналогичным осциллирующим зеркалом с правой стороны от неподвижного. Спецификой служит условие, что период осцилляций зеркала считается много больше времени обхода резонатора излучением. Задача решается методом двух временных масштабов. Этот подход использовался авторами ранее [6-8] для решения нелинейной задачи о движении солитона бозе-эйнштейновского конденсата, заключенного в ловушку с осциллирующими стенками, причем была продемонстрирована достаточно высокая точность результата. Оказалось, что двухмасштабный метод также удобен и для решения рассматриваемой задачи. При указанном выше ограничении естественным образом возникает малый параметр, равный отношению малого периода к большому, так что задача решается методом теории возмущений. В работе найдено нулевое приближение и показано, как непротиворечиво можно построить приближение следующего порядка. Особенностью данного решения является то, что оно справедливо при любой амплитуде колебаний зеркала в интервале от нуля до величины, равной средней длине резонатора. Это позволило показать, что рассматриваемая система может быть использована для эффективной генерации электромагнитного поля. Предложена схема такого генератора. При этом полезно сравнить полученные результаты с результатами других работ, в которых в одномерном случае удалось получить точные решения задачи [3]. Мы обратим внимание лишь на две их них. Это работа [9] и особенно работа [10], где независимо получены точные решения задачи, когда правое зеркало движется равномерно и прямолинейно. В настоящей работе будет показано, что, хотя осциллирующий закон движения существенно отличается от прямолинейного, но существует глубокая аналогия в поведении полной энергии поля в обоих случаях.

Дальнейшее изложение состоит из следующих разделов. В первом из них излагается постановка задачи. 
В следующем изложен метод многих масштабов и дается формулировка задачи в рамках этого метода. Затем приведено решение задачи в нулевом приближении. Далее исследуется возможность получения следующего приближения, а также возможность генерации электромагнитного излучения. В заключительном разделе проводится численный анализ полученных результатов.

\section{Постановка задачи}

В работе исследуется эволюция электромагнитного поля в резонаторе с идеально отражающими металлическими зеркалами, из которых одно покоится (при $x=0$ ), а второе осциллирует около положения своего равновесия, которое соответствует координате $x=L$. Поле в резонаторе (в вакууме) описывается одномерными уравнениями Максвелла для напряженностей электрического $E$ и магнитного $H$ полей:

$$
\begin{aligned}
& \frac{1}{c} \frac{\partial E}{\partial t}+\frac{\partial H}{\partial x}=0 \\
& \frac{1}{c} \frac{\partial H}{\partial t}+\frac{\partial E}{\partial x}=0 .
\end{aligned}
$$

Здесь $c-$ скорость света в вакууме и $t-$ время. При этом движение зеркала описывается уравнением

$$
x_{r}(t)=L+a \sin \omega t
$$

где $\omega$ и $a-$ частота и амплитуда колебаний соответственно.

В данной системе можно выделить два характерных масштаба времени. Первый - это $T_{o s c}$, период осцилляций зеркала, равный

$$
T_{o s c}=\frac{2 \pi}{\omega} .
$$

Второй - это время полного обхода светом эффективного резонатора, у которого подвижное зеркало покоится в положении $x=L$. Очевидно, этот масштаб $T_{p e r}$ будет равен

$$
T_{p e r}=\frac{2 L}{c},
$$

что соответствует круговой частоте $\Omega$

$$
\Omega=\frac{\pi c}{L} \text {. }
$$

Эта частота, очевидно, соответствует частоте основной моды такого резонатора. В данной работе будет исследоваться случай, когда частота осцилляций зеркала много меньше частоты $\Omega$ :

$$
\omega / \Omega=\varepsilon \ll 1 .
$$

В дальнейшем анализ уравнений поля, а также все расчеты удобно проводить, пользуясь безразмерными координатами. В качестве безразмерного времени выберем $\Omega t$, для которого сохраним прежнее обозначение $t$, a в качестве безразмерной координаты $s$ выберем $x$, нормированное на $L / \pi$. Таким образом,

$$
\Omega t \rightarrow t, \quad \frac{\pi x}{L} \rightarrow s
$$

В результате система уравнений (1) переходит в систему вида

$$
\begin{aligned}
& \frac{\partial E}{\partial t}+\frac{\partial H}{\partial s}=0, \\
& \frac{\partial H}{\partial t}+\frac{\partial E}{\partial s}=0 .
\end{aligned}
$$

В свою очередь уравнение для $x_{r}(t)$ переходит в уравнение для $s_{r}(t)$ :

$$
s_{r}(t)=\pi(1+\mu \sin \varepsilon t) .
$$

Здесь $\mu=a / L$. При этом нетрудно показать, что выражению для скорости движения зеркала в новых переменных

$$
\frac{d s_{r}(t)}{d t}=\dot{s}_{r}(t)=\varepsilon \pi \mu \cos \varepsilon t
$$

соответствует исходное выражение $\frac{1}{c} \frac{d x_{r}(t)}{d t}$, где $t$ старая временная переменная.

Рассмотрим теперь начальные и граничные условия, которым должны удовлетворять решения системы (8). В самом общем случае они имеют вид

$$
\begin{aligned}
& \left.E(t, s)\right|_{t=0}=E^{0}(s), \\
& \left.H(t, s)\right|_{t=0}=H^{0}(s), \\
& \left.E(t, s)\right|_{s=0}=0, \\
& \left.\left(E(t, s)-\dot{s}_{r}(t) H(t, s)\right)\right|_{s=s_{r}(t)}=0 .
\end{aligned}
$$

При этом, как следует из третьего уравнения (11) и из первого уравнения (8), начальные условия должны также удовлетворять требованиям

$$
\left.E^{0}(s)\right|_{s=0}=0,\left.\quad \frac{\partial H^{0}(s)}{\partial s}\right|_{s=0}=0 .
$$

Из граничного условия на движущейся границе в свою очередь возникает требование, чтобы начальные условия удовлетворяли соотношению

$$
\left.\left(E^{0}(s)-\dot{s}_{r}(0) H^{0}(s)\right)\right|_{s=s_{r}(0) \equiv \pi}=0 .
$$

Более детально это соотношение будет проанализировано в дальнейшем. Отметим также, что для описания осцилляций зеркала вместо $\sin \omega t$ может быть использована и любая другая регулярная периодическая функция. 


\section{Метод многих масштабов}

Наличие в задаче двух масштабов времени, отношение которых является малой величиной (малый параметр $\varepsilon$ ), указывает на возможность решения задачи методом многих масштабов. Как указывалось выше, ранее этот подход использовался авторами для решения задачи о движении центра тяжести солитона в ловушке с осциллирующими стенками [6-8]. Такое движение описывается уравнением типа уравнения Ньютона, в основе вывода которого лежит нелинейное уравнение Шредингера. Принципиальное отличие рассматриваемой задачи от предыдущих заключается в том, что многомасштабный метод применяется непосредственно к решению уравнений в частных производных, которые зависят не только от временных, но также и пространственных переменных, что усложняет решение задачи.

Введем в соответствии с двумя масштабами задачи (3) и (4) две новых временных переменных: „медленную“ $\tau$ и „быструю“ $\theta$ согласно равенствам

$$
\tau=\varepsilon t, \quad \theta=\vartheta(\tau) / \varepsilon .
$$

В результате производная по времени переходит в сумму производных по новым переменным вида

$$
\frac{\partial}{\partial t}=\vartheta_{\tau} \frac{\partial}{\partial \theta}+\varepsilon \frac{\partial}{\partial \tau}
$$

Подставляя (15) в систему (8), получаем исходную систему уравнений для решения задачи методом многих масштабов. Эта система имеет вид

$$
\begin{aligned}
& \left(\vartheta_{\tau} \frac{\partial}{\partial \theta}+\varepsilon \frac{\partial}{\partial \tau}\right) E+\frac{\partial H}{\partial s}=0, \\
& \left(\vartheta_{\tau} \frac{\partial}{\partial \theta}+\varepsilon \frac{\partial}{\partial \tau}\right) H+\frac{\partial E}{\partial s}=0 .
\end{aligned}
$$

Уравнение движения зеркала при этом удобно представить в форме

$$
\begin{aligned}
& s_{r}(\tau)=\pi(1+q(\tau)), \\
& q(\tau)=\mu \sin \tau,
\end{aligned}
$$

откуда для скорости движения получаем выражение

$$
\frac{d s_{r}(\tau)}{d t}=\varepsilon \pi q_{\tau}(\tau)
$$

Таким образом, с помощью уравнений (16) и (18) в задачу в явном виде вводится малый параметр $\varepsilon$. Поэтому для ее решения поля $E$ и $H$, а также функцию $\vartheta(\tau)$ разумно представить в виде разложения по степеням этого параметра:

$$
\begin{aligned}
& E=E_{0}+\varepsilon E_{1}+\varepsilon^{2} E_{2}+\ldots, \\
& H=H_{0}+\varepsilon H_{1}+\varepsilon^{2} H_{2}+\ldots, \\
& \vartheta(\tau)=\vartheta_{0}(\tau)+\varepsilon \vartheta_{1}(\tau)+\varepsilon^{2} \vartheta_{2}(\tau)+\ldots
\end{aligned}
$$

Если, в свою очередь, подставить эти разложения в уравнения (16), то возникает система рекуррентных уравнений, решая которую можно последовательно определять члены указанных разложений. После подстановки получаем

$$
\begin{gathered}
\vartheta_{0 \tau} \frac{\partial}{\partial \theta} E_{0}+\frac{\partial}{\partial s} H_{0}=0 \\
\vartheta_{0 \tau} \frac{\partial}{\partial \theta} H_{0}+\frac{\partial}{\partial s} E_{0}=0 \\
\vartheta_{0 \tau} \frac{\partial}{\partial \theta} E_{1}+\frac{\partial}{\partial s} H_{1}=-\left(\vartheta_{1 \tau} \frac{\partial}{\partial \theta}+\frac{\partial}{\partial \tau}\right) E_{0}, \\
\vartheta_{0 \tau} \frac{\partial}{\partial \theta} H_{1}+\frac{\partial}{\partial s} E_{1}=-\left(\vartheta_{1 \tau} \frac{\partial}{\partial \theta}+\frac{\partial}{\partial \tau}\right) H_{0}, \\
\vartheta_{0 \tau} \frac{\partial}{\partial \theta} E_{2}+\frac{\partial}{\partial s} H_{2}=-\left[\left(\vartheta_{1 \tau} \frac{\partial}{\partial \theta}+\frac{\partial}{\partial \tau}\right) E_{1}\right. \\
\left.+\vartheta_{2 \tau} \frac{\partial}{\partial \theta} E_{0}\right], \\
\vartheta_{0 \tau} \frac{\partial}{\partial \theta} H_{2}+\frac{\partial}{\partial s} E_{2}=-\left[\left(\vartheta_{1 \tau} \frac{\partial}{\partial \theta}+\frac{\partial}{\partial \tau}\right) H_{1}\right. \\
\left.+\vartheta_{2 \tau} \frac{\partial}{\partial \theta} H_{0}\right] .
\end{gathered}
$$

Эта система решается, как и исходная (8), с учетом начальных и граничных условий, явный вид которых будет выписан ниже.

Нетрудно показать, что начальные условия, фигурирующие в (11), переходят в условия вида

$$
\begin{aligned}
& \left.E_{0}\right|_{\theta=0} ^{\tau=0}=E^{0}(s),\left.\quad H_{0}\right|_{\theta=0} ^{\tau=0}=H^{0}(s), \\
& \left.E_{n}\right|_{\theta=0} ^{\tau=0}=0,\left.\quad H_{n}\right|_{\theta=0} ^{\tau=0}=0, \quad n \geq 1 .
\end{aligned}
$$

Граничное условие для $E$ при $s=0$ переходит в условие

$$
\left.E_{n}\right|_{s=0}=0, \quad n \geq 0 .
$$

Граничное условие на движущемся зеркале в общем случае имеет вид

$$
\left.\left(E(\theta, s ; \tau)-\varepsilon \pi q_{\tau}(\tau) H(\theta, s ; \tau)\right)\right|_{s=s_{r}(\tau)}=0 .
$$

Отсюда следует

$$
\left.E_{0}(\theta, s ; \tau)\right|_{s=s_{r}(\tau)}=0
$$

Для членов разложений с $n \geq 1$ получаем

$$
\left.\left(E_{n}(\theta, s ; \tau)-\pi q_{\tau}(\tau) H_{n-1}(\theta, s ; \tau)\right)\right|_{s=s_{r}(\tau)}=0 .
$$

При $n=1$ это выражение преобразуется в условие

$$
\left.\left(E_{1}(\theta, s ; \tau)-\pi q_{\tau}(\tau) H_{0}(\theta, s ; \tau)\right)\right|_{s=s_{r}(\tau)}=0 .
$$


С другой стороны, продифференцировав выражение (27) по $\theta$ и подставив его в первое из уравнений (20) при $s=s_{r}(\tau)$, получим

$$
\left.\frac{\partial H_{0}}{\partial s}\right|_{s=s_{r}(\tau)}=0 .
$$

Рассмотрим теперь, какие ограничения накладывают полученные соотношения на начальные условия при $\tau=0$ и $\theta=0$ с учетом того, что $s_{r}(0)=\pi$. Из (27) получаем

$$
\left.E^{0}(s)\right|_{s=\pi}=0 .
$$

Поскольку $\left.E_{1}\right|_{\theta=0} ^{\tau=0}=0$ (см. (24)), из (29) следует

$$
\left.H^{0}(s)\right|_{s=\pi}=0 \text {. }
$$

И, наконец, из (30) получаем

$$
\left.\frac{\partial H^{0}}{\partial s}\right|_{s=\pi}=0
$$

Граничные условия (31)-(33), так же как и условия (12), окажутся весьма важными при построении решений рассматриваемой задачи.

\section{Решение задачи в нулевом приближении}

Несложно показать, что решения системы уравнений нулевого приближения (20), удовлетворяющие граничным условиям (25), (12) при $n=0$, в самой общей форме имеют вид

$$
\begin{aligned}
& E_{0}(\theta, s ; \tau)=\alpha(\tau)\left[R_{0}\left(\theta-\vartheta_{0 \tau} s\right)-R_{0}\left(\theta+\vartheta_{0 \tau} s\right)\right], \\
& H_{0}(\theta, s ; \tau)=\alpha(\tau)\left[R_{0}\left(\theta-\vartheta_{0 \tau} s\right)+R_{0}\left(\theta+\vartheta_{0 \tau} s\right)\right] .
\end{aligned}
$$

Здесь $R_{0}$ - искомая функция. В силу однородности системы (20) полученное решение определено с точностью до произвольного множителя $\alpha(\tau)$. Его выражение будет получено ниже.

Потребуем теперь от решения для $E_{0}$, чтобы оно удовлетворяло условию (27) на движущемся зеркале. В результате получаем

$$
R_{0}\left(\theta-\vartheta_{0 \tau} s_{r}(\tau)\right)-R_{0}\left(\theta+\vartheta_{0 \tau} s_{r}(\tau)\right)=0 .
$$

Этому условию нетрудно удовлетворить, если допустить, что $R_{0}(\theta)$ является периодической функцией с периодом $2 \vartheta_{0 \tau} s_{r}(\tau)$. Этот период всегда можно сделать независящим от $\tau$ за счет произвола в выборе функции $\vartheta_{0}(\tau)$. Выберем этот период равным $2 \pi$, тогда

$$
\vartheta_{0 \tau} s_{r}(\tau)=\pi
$$

откуда определяем вид $\vartheta_{0}(\tau)$ (см. (17))

$$
\vartheta_{0}(\tau)=\int_{0}^{\tau} \frac{d \tau^{\prime}}{1+q\left(\tau^{\prime}\right)} .
$$

Следовательно, $\theta=0$ при $\tau=0$.

Таким образом, главная задача заключается в построении $2 \pi$-периодической функции $R_{0}(\theta)$. Для этой цели необходимо воспользоваться начальными условиями. При этом без ограничения общности можно полагать, что $\left.\alpha(\tau)\right|_{\tau=0}=1$. С учетом этого при $\tau=0$ и $\theta=0$ получаем следующие соотношения (см. (34)):

$$
R_{0}(-s)-R_{0}(s)=E^{0}(s), \quad R_{0}(-s)+R_{0}(s)=H^{0}(s) .
$$

Здесь мы воспользовались тем, что

$$
\left.\vartheta_{0 \tau}\right|_{\tau=0}=1
$$

Из (38) можно сразу же определить $R_{0}(s)$ и $R_{0}(-s)$ :

$$
\begin{aligned}
& R_{0}(s)=\frac{1}{2}\left(H^{0}(s)-E^{0}(s)\right), \\
& R_{0}(-s)=\frac{1}{2}\left(H^{0}(s)+E^{0}(s)\right) .
\end{aligned}
$$

Обычно в начальный момент функции $E^{0}(s)$ и $H^{0}(s)$ задаются на промежутке $0 \leq s \leq \pi$, что соответствует промежутку $0 \leq x \leq L$. Именно на этом промежутке и определена функция $R_{0}(s)$ в (40). Для построения периодической функции $R_{0}(s)$ необходимо должным образом продолжить функции $E^{0}(s)$ и $H^{0}(s)$ сначала на промежуток $\pi<x \leq 2 \pi$ (или, что то же самое, на промежуток $-\pi \leq s<0$ ), а затем периодически на всю ось $s$. Заметим, что в данном случае при построении периодического решения безразмерная координата $s$ выполняет ту же роль, что и $\theta$ в (35).

Из (38) следует, что поскольку в левой стороне выражения для $E^{0}(s)$ стоит нечетная по $s$ функция, а для $H^{0}(s)$ - четная, то и продолжение этих функций на промежуток $\pi<s \leq 2 \pi$ очевидно должно быть нечетным и четным соответственно. После этого полученные функции элементарно продолжаются $2 \pi$-периодически на всю ось $s$. Обозначим построенные таким образом $2 \pi$-периодические функции как $E_{p e r}^{0}(s)$ и $H_{p e r}^{0}(s)$. Тогда для периодической функции $R_{0}(s)$, используя $(40)$, окончательно получаем

$$
R_{0}(s)=\frac{1}{2}\left(H_{p e r}^{0}(s)-E_{p e r}^{0}(s)\right) .
$$

Важно отметить, что поскольку функция $E^{0}(s)$ обращается в 0 как при $s=0$, так и при $s=\pi$ (см. (12) и (31)), то антисимметричное продолжение этой функции гарантирует непрерывность в этих точках не только самой функции, но и ее первых производных. Аналогичным образом симметричное продолжение $H^{0}(s)$ при выполнении условий (12), (32), (33) также обеспечивает непрерывность продолжения как самой функции, так и производных. При этом

$$
E_{p e r}^{0}(-s)=-E_{p e r}^{0}(s), \quad H_{p e r}^{0}(-s)=H_{p e r}^{0}(s) .
$$


Соотношения (41) и (34) позволяют построить в явном виде решение задачи в нулевом приближении:

$$
\begin{aligned}
& E_{0}(\theta, s ; \tau)=\frac{1}{2} \alpha(\tau)\left\{\left[H_{p e r}^{0}\left(\theta-\frac{s}{1+q(\tau)}\right)\right.\right. \\
& \left.-E_{p e r}^{0}\left(\theta-\frac{s}{1+q(\tau)}\right)\right]-\left[H_{p e r}^{0}\left(\theta+\frac{s}{1+q(\tau)}\right)\right. \\
& \left.\left.\quad-E_{p e r}^{0}\left(\theta+\frac{s}{1+q(\tau)}\right)\right]\right\}, \\
& H_{0}(\theta, s ; \tau)=\frac{1}{2} \alpha(\tau)\left\{\left[H_{p e r}^{0}\left(\theta-\frac{s}{1+q(\tau)}\right)\right.\right. \\
& \left.\quad-E_{p e r}^{0}\left(\theta-\frac{s}{1+q(\tau)}\right)\right]+\left[H_{p e r}^{0}\left(\theta+\frac{s}{1+q(\tau)}\right)\right. \\
& \left.\left.-E_{p e r}^{0}\left(\theta+\frac{s}{1+q(\tau)}\right)\right]\right\} .
\end{aligned}
$$

Неизвестная функция $\alpha(\tau)$ определяется из условия разрешимости уравнения для поправки первого порядка в регулярных ограниченных функциях. Это будет сделано в следующем разделе.

Сопоставим теперь полученный результат с общим решением системы (8), которое представляет комбинацию двух произвольных функций, одна из которых зависит от разности $(t-s)$, а вторая от суммы $(t+s)$. Легко показать, что в любом интервале времени, заключенном в промежутке

$$
2 \pi N \leq t \leq 2 \pi(N+1),
$$

где $N$ - произвольное целое число, полученное решение (43) с точностью до членов $\sim \varepsilon$ удовлетворяет также и системе уравнений (8).

\section{Определение $\alpha(\tau)$. Возможность генерации электромагнитного поля}

Предлагаемый подход к решению задачи, а также полученное выше решение в нулевом приближении можно считать корректными, если мы в состоянии рассчитать поправку следующего порядка, и эта поправка будет регулярной ограниченной функцией. Условие разрешимости уравнения для поправки первого порядка в таких функциях позволяет при этом определить $\alpha(\tau)$.

Рассмотрим систему уравнений (21) для поправок первого порядка $E_{1}$ и $H_{1}$, которую удобно представить в матричной форме

$$
\vartheta_{0 \tau} \frac{\partial}{\partial \theta} F_{1}+\frac{\partial}{\partial s}\left(\sigma F_{1}\right)=-\left(\vartheta_{1 \tau} \frac{\partial}{\partial \theta}+\frac{\partial}{\partial \tau}\right) F_{0},
$$

где

$$
F_{0}=\left(\begin{array}{c}
E_{0} \\
H_{0}
\end{array}\right), \quad F_{1}=\left(\begin{array}{c}
E_{1} \\
H_{1}
\end{array}\right), \quad \sigma=\left(\begin{array}{ll}
0 & 1 \\
1 & 0
\end{array}\right) .
$$

В случае уравнений, описывающих консервативные системы, условием разрешимости системы уравнений типа (45) в требуемых регулярных функциях является условие ортогональности решения нулевого приближения к правой части уравнения (45), так как левая часть при этом обращается в ноль. В случае открытых систем, каковой является рассматриваемая в настоящей работе, левая часть не обращается в ноль, поскольку появляется внешний источник. Тогда, как мы увидим ниже, условие разрешимости будет представлять уравнение, описывающее баланс между изменением внутренней энергии системы и ее притоком извне (или, наоборот, оттоком). Фактически это уравнение для неизвестной функции $\alpha(\tau)$, из которого мы ее можем определить.

Умножим уравнение (45) слева на транспонированную функцию $F_{0}^{t r}$, которая имеет вид

$$
F_{0}^{t r}=\left(E_{0}, H_{0}\right),
$$

а затем полученное выражение приведем к виду

$$
\begin{aligned}
& \vartheta_{0 \tau} \frac{\partial}{\partial \theta}\left(F_{0}^{t r} F_{1}\right)+\frac{\partial}{\partial s}\left(F_{0}^{t r} \sigma F_{1}\right)-\left\{\vartheta_{0 \tau} \frac{\partial}{\partial \theta} F_{0}^{t r}+\frac{\partial}{\partial s} F_{0}^{t r} \sigma\right\} \\
& \times F_{1}=-\frac{1}{2}\left[\vartheta_{1 \tau} \frac{\partial}{\partial \theta}\left(F_{0}^{t r} F_{0}\right)+\frac{\partial}{\partial \tau}\left(F_{0}^{t r} F_{0}\right)\right] .
\end{aligned}
$$

Очевидно, что выражение в фигурных скобках в левой части (48) обращается в ноль, поскольку совпадает с (20). Оставшееся уравнение усредним по быстрым осцилляциям с периодом $2 \pi$ и проинтегрируем по $s$ от 0 до $\pi(1+q(\tau))$. При этом член с производными по $\theta$ обращается в ноль. Теперь, разделив оставшиеся интегралы на $4 \pi$, нетрудно получить следующее соотношение:

$$
\frac{\partial}{\partial \tau} W_{0}=-J_{0},
$$

где

$$
\begin{aligned}
& W_{0}=\frac{1}{2 \pi} \int_{0}^{2 \pi} d \theta \int_{0}^{\pi(1+q(\tau))} d s \frac{\left(E_{0}^{2}+H_{0}^{2}\right)}{8 \pi}, \\
& J_{0}=\pi q_{\tau} \frac{1}{2 \pi} \int_{0}^{2 \pi} d \theta \frac{\left.H_{0}^{2}\right|_{s=\pi(1+q(\tau))} .}{8 \pi} .
\end{aligned}
$$

Здесь $W_{0}$ - это усредненная по быстрым осцилляциям энергия поля в резонаторе, а $J_{0}-$ поток энергии, связанный с движением зеркала и пропорциональный его скорости $\pi q_{\tau}$. Отметим, что при выводе (49) было учтено также условие (27).

Для вычисления интегралов, входящих в (50), воспользуемся формулами (34), которые дают решение задачи в наиболее общей форме. В результате для $W_{0}$ 


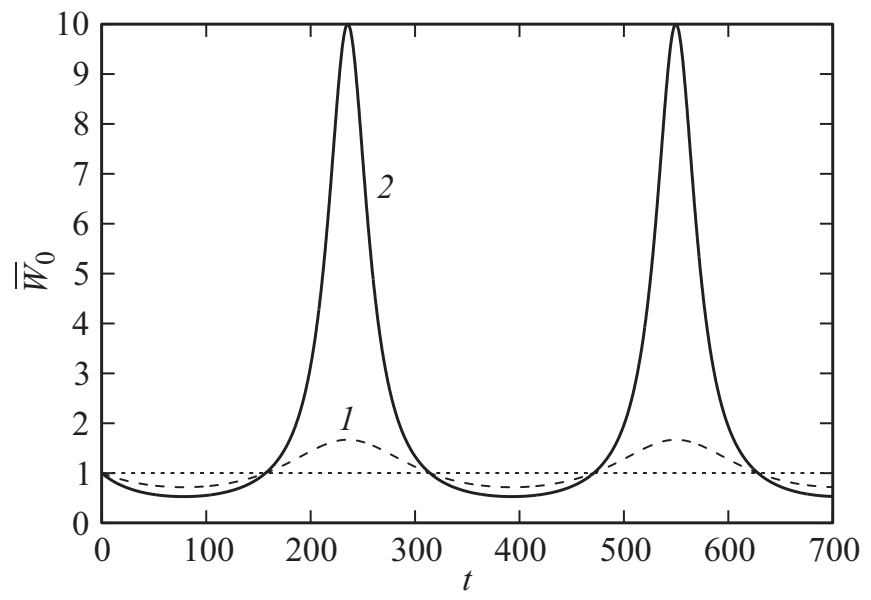

Рис. 1. Временная зависимость нормированной усредненной энергии поля в резонаторе; $\mu=0.4$ (пунктирная линия 1 ) и $\mu=0.9$ (сплошная линия 2), $\varepsilon=0.02$.

и $J_{0}$ получаем выражения вида

$$
\begin{aligned}
& W_{0}=(1+q(\tau)) \alpha^{2}(\tau) \frac{1}{4 \pi} \int_{-\pi}^{\pi} R_{0}^{2}(s) d s, \\
& J_{0}=q_{\tau} \alpha^{2}(\tau) \frac{1}{4 \pi} \int_{-\pi}^{\pi} R_{0}^{2}(s) d s .
\end{aligned}
$$

Подставляя эти выражения в (49) и решая полученное уравнение с начальным условием $\left.\alpha(\tau)\right|_{\tau=0}=1$, получаем

$$
\alpha(\tau)=(1+q(\tau))^{-1} .
$$

Вычисление интеграла в (51) на основе начальных условий приводит к выражению для энергии электромагнитного поля в резонаторе в начальный момент времени $\Sigma^{0}$ :

$$
\Sigma^{0}=\frac{1}{4 \pi} \int_{-\pi}^{\pi} R_{0}^{2}(s) d s=\int_{0}^{\pi} d s \frac{\left(E^{0}(s)\right)^{2}+\left(H^{0}(s)\right)^{2}}{8 \pi} .
$$

С учетом (34) можно записать нормированную энергию поля в резонаторе $\bar{W}_{0}=\left(W_{0} / \Sigma^{0}\right)$ в виде

$$
\bar{W}_{0}(t)=(1+\mu \sin (\varepsilon t))^{-1} \text {. }
$$

На рис. 1 представлены графики $\bar{W}_{0}$ как функции времени $t$ при $\mu=0.4$ и 0.9. Видно, что энергия поля в резонаторе периодически меняется обратно пропорционально мгновенной длине резонатора. Как можно объяснить подобные колебания энергии? Очевидно, что имеющееся поле в резонаторе наводит зеркальное изображение своего распределения на металлическом зеркале этого резонатора. В свою очередь движение такого зеркала приводит к генерации поля, которое при сближении зеркал находится в фазе с полем резонатора и увеличивает его, а при расхождении зеркал находится с ним в противофазе и уменьшает энергию поля.

Существенное увеличение первоначальной энергии в резонаторе при малых расстояниях между зеркалами указывает на возможность использования такой системы в качестве как усилителя (с одновременным преобразованием частоты), так и генератора электромагнитного излучения. Можно рассмотреть простейший вариант их чисто теоретической схемы. Пусть в момент $t=0$ подвижное зеркало в резонаторе, в котором отсутствует поле, максимально удалено от неподвижного. В этот же момент в резонатор закачивается поле с энергией $W_{i n}$. Тогда энергия поля в этом резонаторе будет описываться формулой

$$
\Delta W_{0}=\frac{(1+\mu) W_{i n}}{1+\mu \sin \left[\varepsilon\left(t+\frac{\pi}{2 \varepsilon}\right)\right]} .
$$

Когда зеркала сближаются на минимальное расстояние, энергия в соответствии с (55) достигает максимума, равного $\Delta W_{0}=\frac{(1+\mu) W_{i n}}{1-\mu}$. В варианте усилителя в резонатор электромагнитные импульсы впрыскиваются периодически в момент максимальной длины резонатора и выводятся в момент минимальной длины. Для генератора внешнее излучение не требуется, но выводимая при минимальной длине резонатора энергия попадает на делитель, от которого часть энергии, равная $W_{i n}$, отводится в цепь обратной связи и в момент максимального расхождения зеркал закачивается обратно в резонатор. В результате получаем постоянно действующий генератор с потоком электромагнитной энергии на выходе

$$
S=\frac{\omega}{2 \pi}\left[\frac{1+\mu}{1-\mu}-1\right] W_{i n}=\frac{\omega}{\pi} \frac{\mu}{1-\mu} W_{i n} .
$$

Так, при $\mu=0.9$, как видно из (56), энергия резонатора на выходе за один период колебаний зеркала увеличивается в 18 раз. Разумеется, существуют различные варианты отвода выходной энергии генератора и построения его схемы. Очевидно также, что приведенная схема генератора является идеализированной и не учитывает разнообразные потери, которые возникают в реальных условиях. Но теоретическая возможность сколько угодно близкого подхода зеркал друг к другу, когда энергия многократно возрастает, позволяет надеяться, что выигрыш по энергии возможен, а следовательно, возможна и генерация энергии. Периодическое повышение частоты излучения на каждом цикле генерации будет ограничиваться дисперсией зеркал резонатора и другими не учитываемыми здесь факторами.

Необходимо отметить, что возможность генерации электромагнитного излучения при движении зеркал резонатора изучалась и ранее. Так, в работе [11] рассматривалась схема взрывомагнитного генератора энергии, в которой генерация возникала при схлопывании 
оболочки резонатора за счет взрыва. Другой вариант генерации электромагнитной энергии связан с возможностью увеличения энергии кванта основной моды резонатора вследствие сдвига частоты излучения в большую сторону за счет эффекта Допплера при периодическом отражении от осциллирующего зеркала резонатора (см. [1-5] и соответствующие ссылки). В предлагаемой нами схеме генерация электромагнитной энергии осуществляется периодически и без разрушения резонатора за счет приращения энергии поля при сближении зеркал.

Представляет интерес сравнить зависимость изменения энергии поля резонатора от положения подвижного зеркала резонатора, полученную в нашем решении, с зависимостями, полученными в точных решениях. Мы ограничимся сравнением с работой [10], в которой найдено точное решение для случая, когда зеркало движется равномерно и прямолинейно. Элементарное рассмотрение показывает, что, как и в нашем случае, энергия в точном решении обратно пропорциональна текущей длине резонатора. Таким образом, зависимость изменения энергии от длины резонатора является достаточно общей независимо от характера движения зеркала.

Когда расстояние между зеркалами стремится к нулю, выражение для энергии поля в резонаторе формально расходится. Разумеется, это связано с тем, что рассматриваемая схема является идеализированной. В такой схеме прежде всего необходимо учесть дисперсию зеркал. Поскольку при сближении зеркал характерные частоты резонатора неограниченно возрастают, а сами зеркала при любых частотах не являются идеальными, то в реальности излучение, частично поглощаясь, будет, когда частоты станут предельно большими, просто пронизывать зеркала, так что энергия поля в резонаторе будет всегда конечной.

Но даже в случае идеально отражающих зеркал существует механизм, который ограничивает сближение зеркал, а следовательно, и рост энергии. Этим механизмом является световое давление, которое, однако, начинает играть заметную роль лишь при крайне высокой интенсивности электромагнитного поля.

\section{Численный анализ динамики поля в резонаторе}

Рассмотрим динамику поля в резонаторе, выбрав конкретные начальные условия для полученного решения (43). Для упрощения расчетов выберем следующие функции:

$$
E^{0}(s)=e^{0} \sin s, \quad H^{0}(s)=h^{0} \sin ^{2} s, \quad 0 \leq s \leq \pi .
$$

Такой выбор начального условия позволяет реализовать изложенную выше процедуру построения решения, так как эти функции могут быть естественным образом продолжены с периодом $2 \pi$ и за пределы

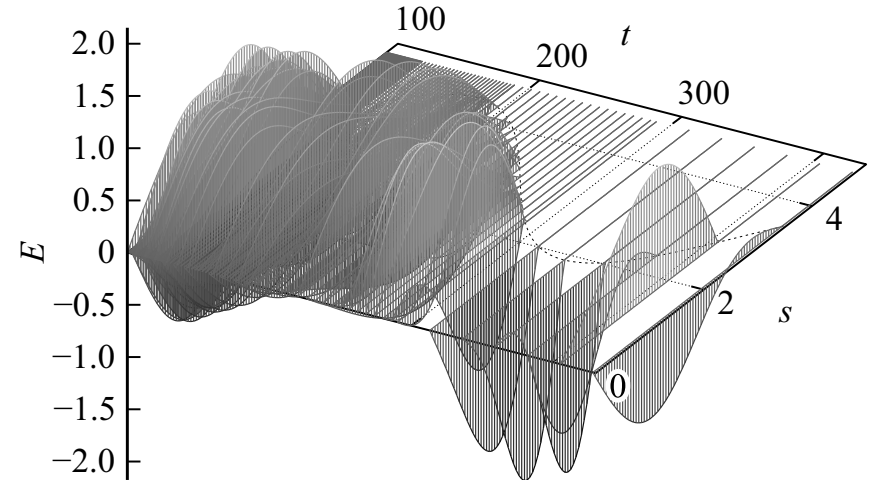

Рис. 2. Напряженность поля $E(t, s)$ на одном периоде в резонаторе; $\beta=-1 ; \mu=0.4$. Пунктирная синусоида здесь и далее указывает область внутри динамического резонатора.

области существования начальных условий. Тогда эти функции превращаются в функции $E_{p e r}^{0}(s)$ и $H_{p e r}^{0}(s)$. При этом, как легко убедиться, функции (57) удовлетворяют всем требуемым граничным условиям (12), а также (31)-(33). В результате решение задачи с начальными условиями (57) будет иметь вид (см. (43))

$$
\begin{aligned}
& E(\theta, s ; \tau)=\frac{1}{2} \alpha(\tau)\left\{\left[\beta \sin ^{2}\left(\theta-\frac{s}{1+q(\tau)}\right)\right.\right. \\
& \left.-\sin \left(\theta-\frac{s}{1+q(\tau)}\right)\right]-\left[\beta \sin ^{2}\left(\theta+\frac{s}{1+q(\tau)}\right)\right. \\
& \left.\left.-\sin \left(\theta+\frac{s}{1+q(\tau)}\right)\right]\right\}, \\
& H(\theta, s ; \tau)=\frac{1}{2} \alpha(\tau)\left\{\left[\beta \sin ^{2}\left(\theta-\frac{s}{1+q(\tau)}\right)\right.\right. \\
& \left.-\sin \left(\theta-\frac{s}{1+q(\tau)}\right)\right]+\left[\beta \sin ^{2}\left(\theta+\frac{s}{1+q(\tau)}\right)\right. \\
& \left.\left.-\sin \left(\theta+\frac{s}{1+q(\tau)}\right)\right]\right\} .
\end{aligned}
$$

В этих формулах (см. (43), (57))

$$
E=E_{0} / e^{0}, \quad H=H_{0} / e^{0}, \quad \beta=h^{0} / e^{0} .
$$

При проведении расчетов мы переходим к единому времени $t$ вместо $\theta$ и $\tau$ по формулам

$$
\begin{gathered}
\tau=\varepsilon t, \\
\theta=\int_{0}^{t} \frac{d t^{\prime}}{1+\mu \sin \left(\varepsilon t^{\prime}\right)} \\
=\frac{2}{\sqrt{1-\mu^{2}}} \operatorname{Arctg} \frac{\sqrt{1-\mu^{2}} \operatorname{tg}(t / 2)}{1+\mu \operatorname{tg}(t / 2)} .
\end{gathered}
$$



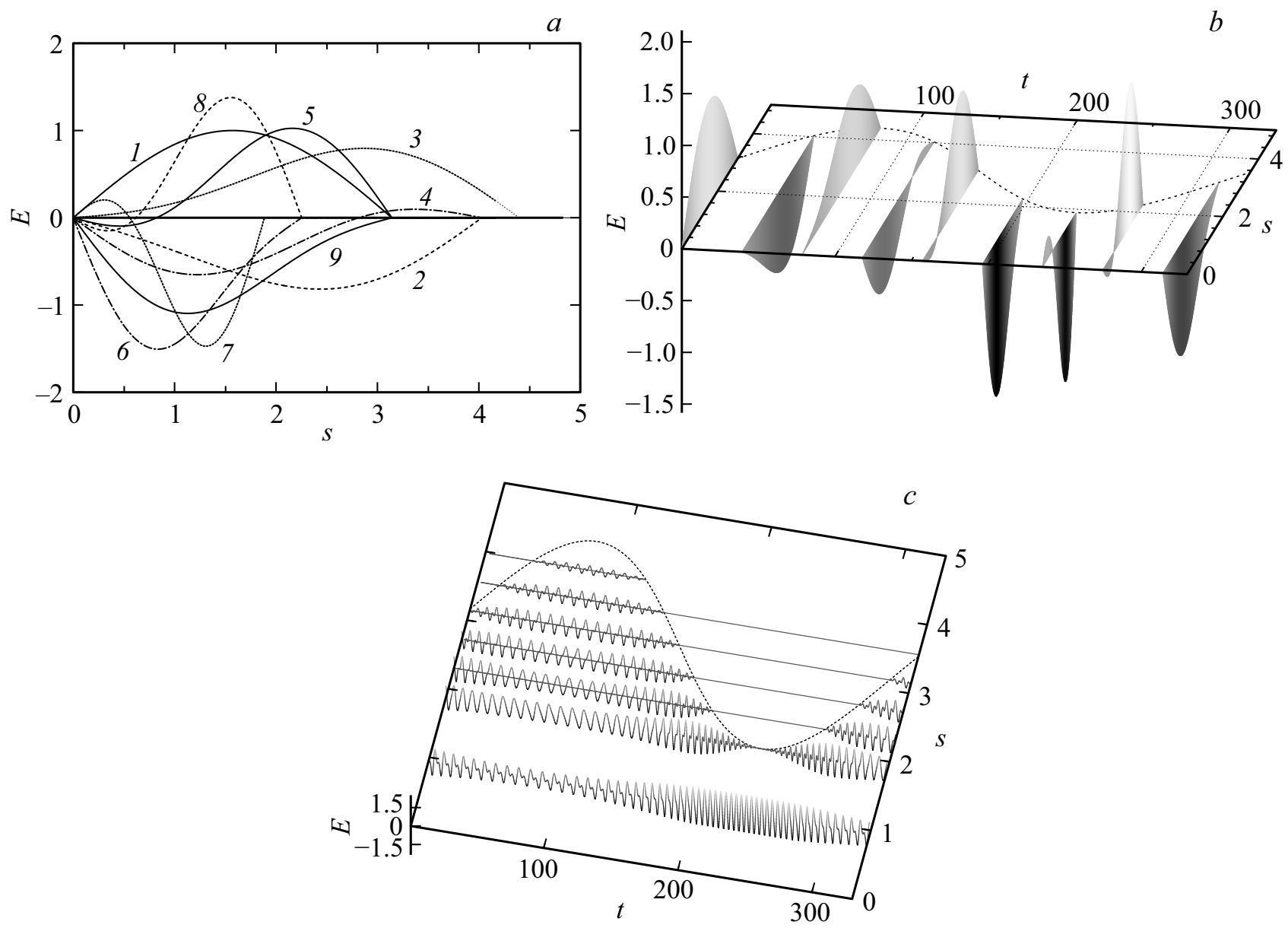

Рис. 3. (а) Совокупный набор разрезов трехмерного графика по оси $t: 1-t=0,2-t=\frac{\pi}{4 \varepsilon}, 3-t=\frac{\pi}{2 \varepsilon}, 4-t=\frac{3 \pi}{4 \varepsilon}, 5-$ $t=\frac{\pi}{\varepsilon}, 6-t=\frac{5 \pi}{4 \varepsilon}, 7-t=\frac{3 \pi}{2 \varepsilon}, 8-t=\frac{7 \pi}{4 \varepsilon}, 9-t=\frac{2 \pi}{\varepsilon}$. (b) Набор разрезов по оси $t$ на трехмерном графике. (c) Набор разрезов по оси $s ; \mu=0.4 ; \beta=1$.

Конкретные вычисления будут проведены для функции $E(\theta, s ; \tau) \equiv E(t, s)$. На рис. 2 изображен трехмерный график этой функции на одном периоде колебаний зеркала. Более наглядную информацию о данной функции можно получить, изобразив разрезы трехмерных графиков по осям $t$ и $s$ при разных значениях параметров $\beta$ и $\mu$. Значение $\varepsilon$ во всех расчетах одинаково и равно $\varepsilon=1 / 50$. Значения $t$, при которых сделаны разрезы, указаны на графиках. Что касается значений, соответствующих разрезам по оси $s$, то они следующие:

$$
\begin{gathered}
s=\frac{1}{2} \pi(1-\mu) ; s=\pi(1-\mu) ; s=\pi\left(1-\frac{2}{3} \mu\right) ; \\
s=\pi\left(1-\frac{1}{3} \mu\right) ; s=\pi ; s=\pi\left(1+\frac{1}{3} \mu\right) ; \\
s=\pi\left(1+\frac{2}{3} \mu\right) .
\end{gathered}
$$

На всех графиках переменные $t$ и $s$ заключены в пределах $0 \leq t \leq 2 \pi / \varepsilon$ и $0 \leq s \leq \pi$.
На рис. 3 приведены три графика разрезов по осям $t$ и $s$. Первые два графика (разрезы по оси $t$ ) наглядно демонстрируют изменение формы импульса в зависимости от $s$. На первый взгляд, картина сжатия импульсов похожа на сжатие обычного изолированного импульса. Однако на самом деле высота импульса существенно больше, чем при обычном сжатии, поскольку энергия импульса также возрастает. На последнем графике также наглядно продемонстрированы мелкомасштабные осцилляции поля по времени на фоне крупномасштабных осцилляций зеркала. Все эти графики совместно демонстрируют достаточно сложную динамику поля в резонаторе как в пространстве, так и во времени.

На рис. 4 приведен такой же набор графиков, но при значительно большей амплитуде колебаний зеркала, соответствующей $\mu=0.9$. Нетрудно видеть, что, когда расстояние между зеркалами минимально, высота импульсов и соответственно их интенсивность и энергия резко возрастают (см. линию 7 на рис. 4, $a$ ). Так, в схеме генератора, обсуждавшийся выше, изме- 

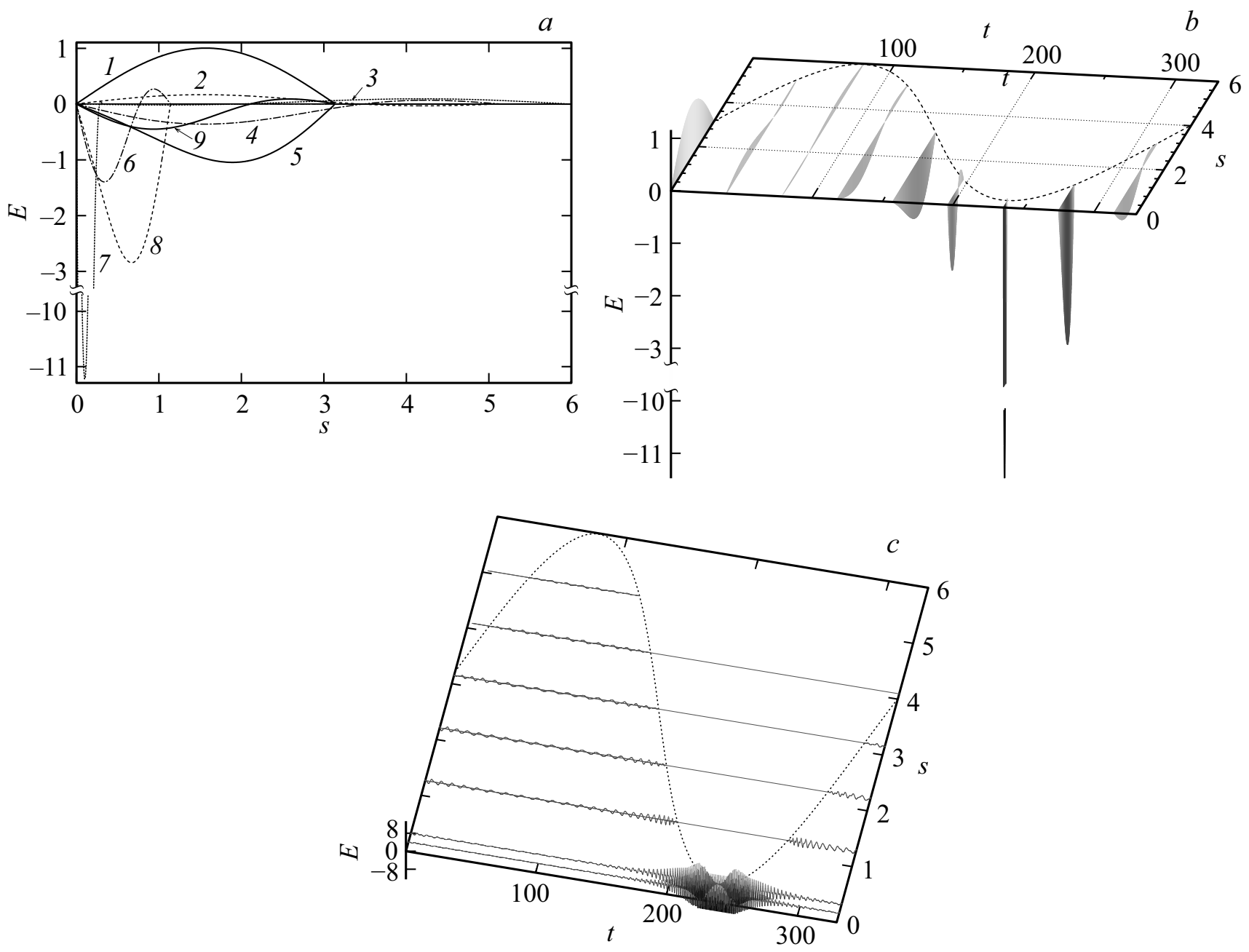

Рис. 4. Тоже, что на рис. 3 , при $\mu=0.9 ; \beta=1$.

нение базы резонатора от максимальной до минимальной увеличивает энергию поля резонатора при $\mu=0.9$ в $\frac{1+\mu}{1-\mu}=19$ раз. Сравнение графиков $c$ на обоих рисунках показывает, что при $\mu=0.9$ в области максимального сближения зеркал происходит взрывной рост частоты колебаний поля. Это специально проиллюстрировано на рис. 5.

Как видно из приведенных графиков, напряженность поля одного и того же импульса вдоль осевой координаты $s$ в фиксированный момент времени $t$ может быть как положительной, так и отрицательной. Это связано с интерференцией импульсов электрического и магнитного полей. Если уменьшать начальный вклад того или другого поля, то огибающие импульсов становятся более симметричными. На рис. 6 приведены огибающие импульсов вдоль оси $s$, когда начальный вклад $H^{0}$ от магнитного поля равен нулю $(\beta=0)$ при $\mu=0.4$. Наглядно видно, что импульсы электрического поля совершенно симметричны, а их огибающие целиком располагаются либо только в области положи- тельных значений напряженности электрического поля, либо только в отрицательной. Ту же тенденцию можно наблюдать и на рис. 7 при $\mu=0.9$. Что касается разрезов вдоль оси $t$, то поведение огибающих напряженности поля аналогично поведению на предыдущих рисунках и сохраняет те же тенденции.

В заключение следует отметить, что исследованная динамика поля в резонаторе соответствует, вообще говоря, конкретному выбору начальных условий. И хотя она отражает определенные общие закономерности, тем не менее другие начальные условия могут заметно изменить наблюдаемую картину.

\section{Заключение}

На основе проведенных исследований необходимо отметить следующие из полученных результатов, а также выделить основные выводы настоящей работы.

На основе метода многих масштабов развит математический подход к решению уравнений Максвелла, 

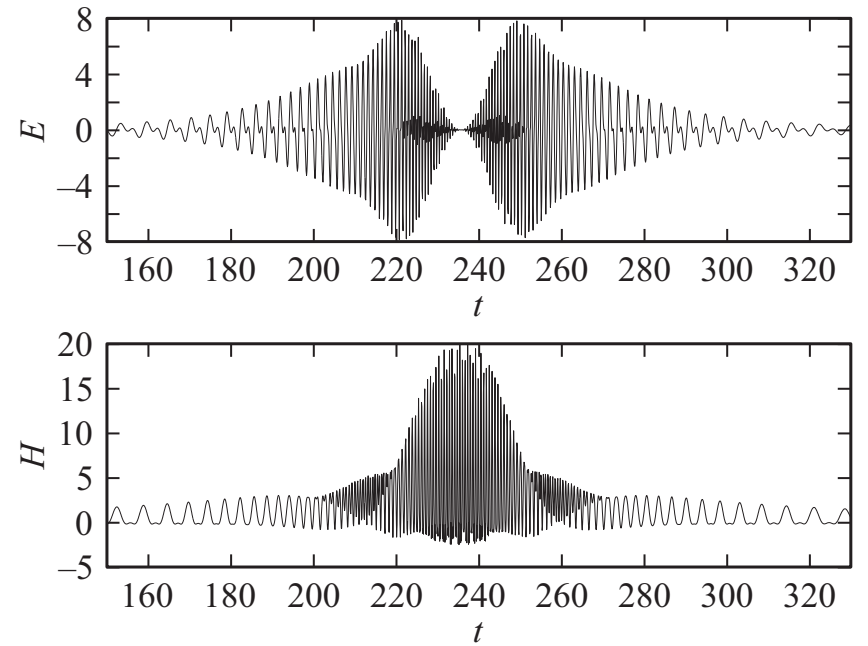

Рис. 5. Разрезы трехмерных графиков полей $E(t, s)$ и $H(t, s)$ по оси $s$ при $s=\pi(1-\mu)$ в области минимального расстояния между зеркалами, $\mu=0.9, \beta=1$.

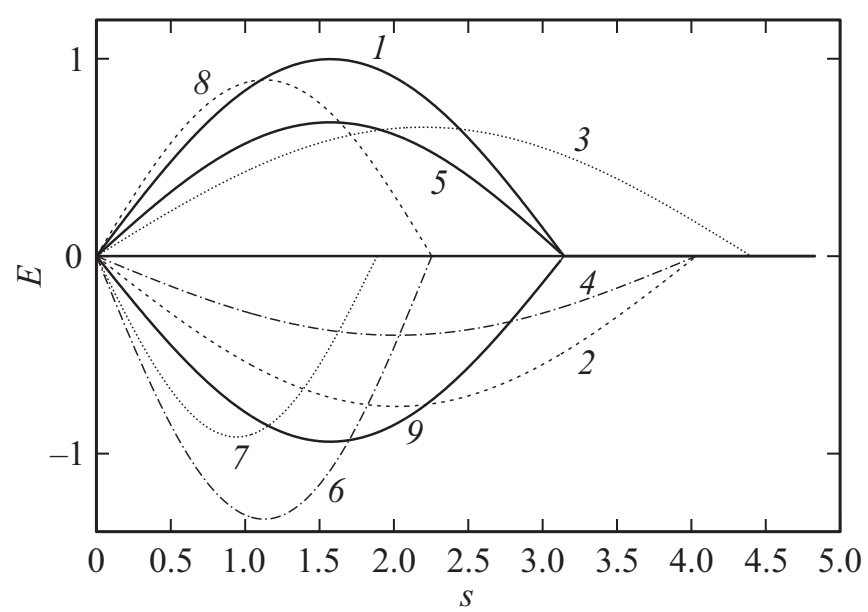

Рис. 6. Совокупный набор разрезов трехмерного графика по оси $t ; \mu=0.4 ; \beta=1$ (соответствует значениям $t$ на рис. 3 ).

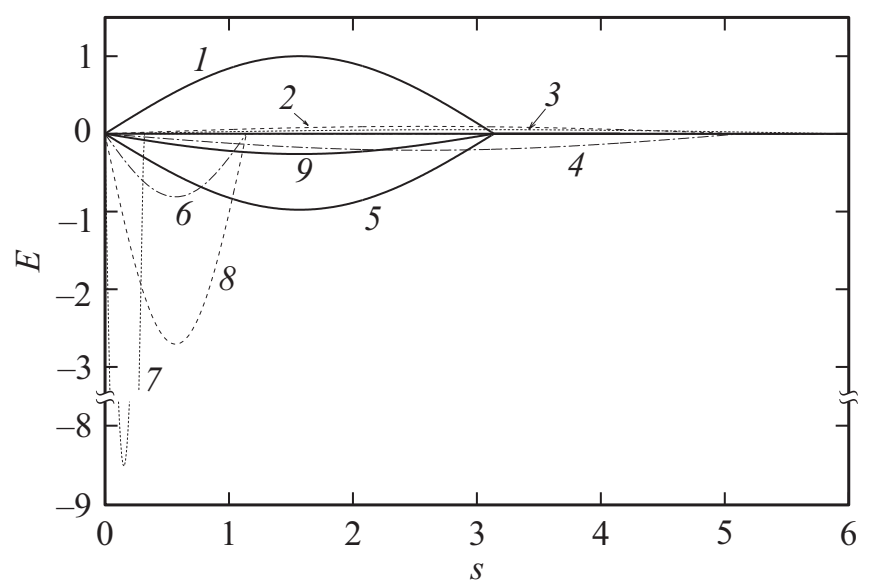

Рис. 7. Совокупный набор разрезов трехмерного графика по оси $t ; \mu=0.9 ; \beta=1$ (нумерация линий соответствует значениям $t$ на рис. 3). описывающих динамику поля в одномерном двухзеркальном резонаторе с неподвижным и осциллирующим металлическими зеркалами. Исследован случай, когда период колебаний зеркала много больше времени полного прохода света через резонатор. Это позволило решить задачу аналитически.

Обнаружено, что усредненная по мелкомасштабным временным осцилляциям энергия поля в резонаторе обратно пропорциональна текущей длине резонатора и, таким образом, изменяется с частотой осцилляций этого зеркала. Сравнение зависимости изменения энергии поля в резонаторе, полученной на основе точного решения задачи для случая равномерного и прямолинейного движения зеркала [10], приводит к аналогичному результату. Таким образом, такого рода зависимость является достаточно общей независимо от закона движения зеркала.

Из сказанного следует, что при сближении зеркал возрастает не только интенсивность поля, но и его энергия, а также его мгновенная частота. Соответственно при расхождении зеркал эти величины убывают. Эти колебания энергии поля можно объяснить тем, что движущееся в поле резонатора металлическое зеркало само генерирует электромагнитное поле. При движении навстречу другому зеркалу генерируемое поле и поле в резонаторе находятся в фазе и усиливают друг друга, в результате чего общая энергия возрастает. При расхождении зеркал фаза генерируемого поля меняется на противоположную, и оба поля ослабляют друг друга. При этом, чем больше амплитуда колебаний подвижного зеркала, тем больше перепады энергии при колебаниях.

При сближении зеркал энергия поля может неограниченно возрастать. Это связано с тем, что в работе рассматривается весьма идеализированная схема резонатора. Проведен анализ факторов, ограничивающих рост поля в резонаторе при сближении зеркал с учетом реальных условий.

Показано, что рассмотренная система может быть использована для генерации электромагнитного поля. Приведена возможная схема такого генератора. Выполнены численные расчеты полей для конкретных начальных условий, подтверждающие указанные выше выводы.

\section{Список литературы}

[1] Красильников В.Н. Параметрические волновые явления в классической электродинамике. Л.: Изд-во ЛГУ, 1996. $299 \mathrm{c}$.

[2] Розанов Н.Н., Мацковский А.А., Малевич В.Л., Синищын Г.В. // Опт. и спектр. 2015. Т. 119. № 1. C. 93-95. Rosanov N.N., Matskovskii A.A., Malevich V.L., Sinitsyn G.V. // Optics and Spectroscopy. 2015. V. 119. N 1. P. 89-91.

[3] Розанов Н.Н., Федоров Э.Г. // Опт. и спектр. 2016. Т. 120. № 5. C. 855-859; Rosanov N.N., Fedorov E.G. // Opt. Spectrosc. 2016. V. 120. N 5. P. 803-807. 
[4] Розанов Н.Н., Федоров Э.Г., Мацковский А.А. // Квант. электрон. 2016. Т. 46. № 1. С. 13-15; Rosanov N.N., Fedorov E.G., Matskovsky A.A. // Quant. Electron. 2016. V. 46. N 1. P. 13-15.

[5] Dodonov V.V., Klimov A.B. // Phys. Rev. A. 1996. V. 53. N 4. P. 2664-2682.

[6] Веретенов Н.А., Высотина Н.В., Нестеров Л.А., Розанов Н.Н. // Опт. и спектр. 2015. Т. 119. № 5. С. 800-809. Veretenov N.A., Vysotina N.V., Nesterov L.A., Rosanov N.N. // Opt. and Spectrosc. 2015. V. 119. N 5. P. 822-831.

[7] Веретенов Н.А., Высотина Н.В., Нестеров Л.А., Розанов Н.Н. // ОПт. и спектр. 2016. Т. 120. № 1. С. 135-148. Veretenov N.A., Vysotina N.V., Nesterov L.A., Rosanov N.N. // Opt. and Spectrosc. 2016. V. 120. N 1. P. 120-133.

[8] Веретенов Н.А., Нестеров Л.А., Розанов Н.Н. // Опт. и спектр. 2016. Т. 120. № 6. С. 998-1004. Veretenov N.A., Nesterov L.A., Rosanov N.N. // Opt. and Spectrosc. 2016. V. 120. N 6. P. 933-939.

[9] Balazs N.L. // J. Math. Anal. Appl. 1962. V. 5. N 2. P. 346.

[10] Баранов Р.И., Широков Ю.М. // ЖЭТФ 1967. Т. 53. C. 2123-2130; Baranov R.I., Shirokov Yu.M. // Sov. Phys. JETP. 1968. V. 26. N 6. P. 1199-1202.

[11] Сахаров А.Д. // УФН. 1966. Т. 88. № 4. С. 725. 